\title{
Immune adaptor protein SKAP1 (SKAP-55) forms homodimers as mediated by the $\mathrm{N}$-terminal region
}

\author{
Monika Raab ${ }^{1}$, Klaus Strebhardt ${ }^{1,2}$ and Christopher E. Rudd ${ }^{1,3,4^{*}}$ (D)
}

\begin{abstract}
Objective: Immune cell adaptor protein SKAP1 couples the antigen-receptor (TCR/CD3) with the activation of LFA-1 adhesion in T-cells. Previous work by ourselves and others have shown that SKAP1 can directly bind to other adaptors such as ADAP and RapL. However, it has been unclear whether SKAP1 can form homodimers with itself and the regions within SKAP1 that mediated homodimer formation.

Results: Here, we show that SKAP1 and SKAP2 form homodimers in cells. Homodimer formation of immune adaptor protein SKAP1 (SKAP-55) are mediated by residues A17 to L21 in the SKAP1 N-terminal region. SKAP1 dimer formation was not needed for its binding to RapL. These data indicate that the pathway linking SKAP1 to RapL is not dependent on the homo-dimerization of SKAP1.
\end{abstract}

Keywords: SKAP1, T-cells, Dimer, RapL

\section{Introduction}

T-cells are activated by a combination of protein-tyrosine kinases and adaptor proteins which mediate the formation of multi-protein complexes $[1,2]$. Immune cell adaptors regulate $\mathrm{T}$-cell proliferation and function [1-3]. SLP-76 (SH2 domain containing leukocyte protein of $76 \mathrm{kDa}$ ) is one such adaptor that is needed for phospholipase $\mathrm{C} \gamma 1$ ( $\mathrm{PLC} \gamma 1$ ) activation, calcium mobilization and thymic differentiation $[4,5]$. It has an $\mathrm{N}$-terminal sterile- $\alpha$ motif (SAM) and a carboxy-terminal SH2 domain that binds to adhesion and degranulation-promoting adapter protein (ADAP) $[6,7]$ and the hematopoietic progenitor kinase-1 (HPK-1) [8]. The C-terminal SH2 domain SLP-76 binds to the ADAP $[6,7,10]$, while ADAP in turn binds to SKAP-1 [10, 11]. SKAP-1 is an adaptor with a unique $\mathrm{N}$ terminus, a $\mathrm{PH}$ domain and a $\mathrm{C}$ terminal $\mathrm{SH} 3$ domain $[9,10]$. SKAP1 SH3 domain binds to proline residues in ADAP while the ADAP-SH3-like domain binds to

*Correspondence: christopher.e.rudd@umontreal.ca

${ }^{4}$ Cell Signalling Section, Department of Pathology, Cambridge University, Cambridge CB2 1QP, UK

Full list of author information is available at the end of the article
SKAP1 $[13,14]$. SKAP1 couples the TCR to the activation of LFA-1 [11-17]. SKAP1 regulates RapL-Rap1 binding induced by antigen-receptor ligation [16-18]. SKAP55 dimer formation has been shown by imaging studies to stabilize SLP-76 micro-clusters and facilitate adhesion [19].

In this study, we have assessed whether SKAP1 and SKAP2 can form homodimers and the region involved in the dimerization. We show biochemically that SKAP1 and SKAP2 can form homodimers in the generation of signals in T-cells. Homodimer formation of immune adaptor protein SKAP1 (SKAP-55) are mediated by the $\mathrm{N}$-terminal region.

\section{Main text \\ Methods \\ Cell culture}

293T cells were grown in DMEM culture medium with $10 \%$ fetal calf serum (FCS), $2 \mathrm{mM}$ L-glutamine, penicillin, and streptomycin. 


\section{Antibodies}

Antibodies to GFP and GST were from Santa Cruz. Anti-SKAP1 (BD Transduction Laboratories), anti-V5 (Invitrogen), anti-FLAG and anti- $\beta$-actin (Sigma) were purchased as assigned. HRP-conjugated secondary antibodies (1:5000) were from Santa Cruz and biotinylated secondary antibodies were from DAKO.

\section{Constructs and transfection}

The constructs of SKAP1 and SKAP2 were inserted into a pGEX5x-3 (GE Healthcare) and into a $3 \times$ Flag-tagged as well as the vectors encoding EGFP-tagged pcDNA3.1Hygro (Invitrogen). Site-directed mutagenesis was conducted using QuickChange protocol and Pfu Ultra II Fusion HS DNA Polymerase (Stratagene). Transfections were conducted using BTX ECM 830 electroporator as described [18].

\section{Immunoblotting}

Precipitations were conducted by lysis in Triton X-100 lysis buffer followed by the incubation with antibody for $1-2 \mathrm{~h}$ at $4{ }^{\circ} \mathrm{C}$ and purification of complexes using protein G-Sepharose beads (10\% w/v) as described [16-18]. For blotting, material on gels transferred onto nitrocellulose filters (Schleicher and Schuell) and detected using horseradish peroxidase-conjugated rabbit anti-mouse antibody together with enhanced chemiluminescence (ECL, Amersham Biosciences).

\section{GST pull down assay}

The expression of recombinant GST-proteins was induced in Escherichia coli BL21 cells at $37^{\circ} \mathrm{C}$ for $2 \mathrm{~h}$ by the addition of $1 \mathrm{mM}$ IPTG. GST-fused proteins were purified with the Cell Lytic B protocol (Sigma \#B7435). Cell lysates were incubated with GST fusion proteins for $3 \mathrm{~h}$ followed by analysis in SDS-PAGE and western blotting as described [18].

\section{Results}

\section{SKAP1 binds to SKAP1 and SKAP2}

To assess whether SKAP1 can interact with itself and SKAP2, each was expressed in $293 \mathrm{~T}$ cells followed by precipitation with anti-Flag (Fig. 1). Cell lysates or precipitates were then blotted with anti-Flag or GFP. Flag-tagged SKAP1 and GFP-tagged SKAP1 were co-expressed followed by precipitation with anti-Flag. Anti-Flag precipitated Flag-tagged SKAP-1 as well as GFP-tagged SKAP1 from cell lysates (lane 5). This indicates that SKAP1 could form homodimers with itself. Similarly, Flag-tagged SKAP2 and GFP-tagged SKAP2 were co-expressed followed by precipitation with anti-Flag. Anti-Flag precipitated Flag-tagged SKAP2 as well as GFP-tagged SKAP2
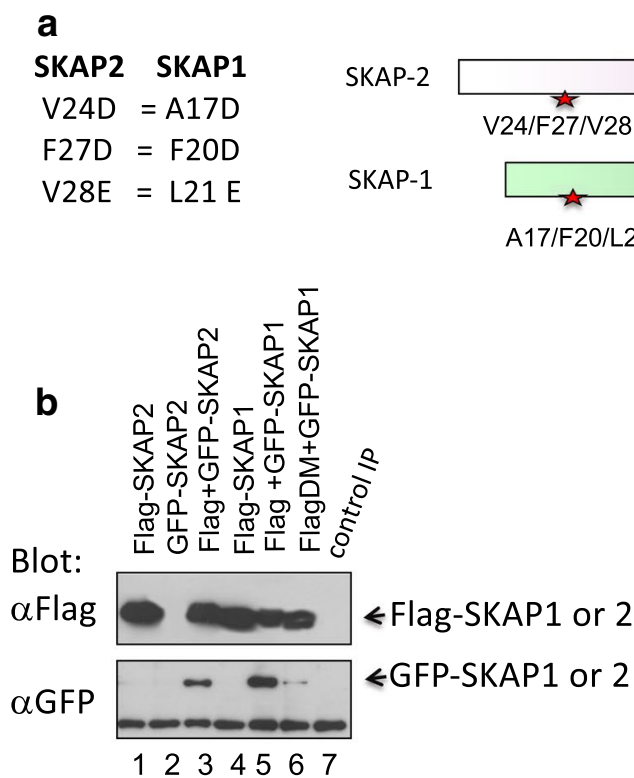

Anti-Flag IP

SKAP-2

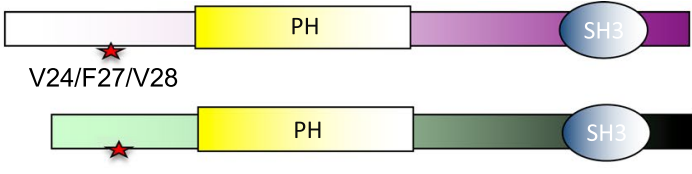

A17/F20/L21

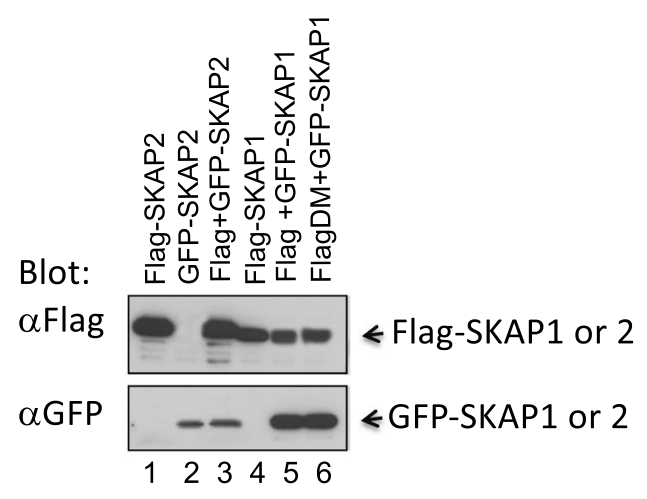

Lysate

Fig. 1 SKAP1 and SKAP2 form homodimers. a Model of the structure of SKAP1 and SKAP2. Mutations in the dimerization domain of SKAP-1 A17/ F20/L21 is shown. $\mathbf{b}$ Co-precipitation of SKAP1 with SKAP-1 and SKAP2 with SKAP2. Left panel: anti-FLAG was used to precipitate antigen from lysates of transfected 293T cells followed by blotting with anti-FLAG or anti-GFP. Lane 1: Flag-SKAP2; lane 2: GFP-SKAP2; lane 3: Flag-SKAP2 and GFP-SKAP2; lane 4: Flag-SKAP1; lane 5: Flag and GFP-SKAP1; lane 6: FlagDM (A17/F20/L21) and GFP-SKAP1. Right panel: blotting of cell lysates from transfected 293T cells seen in left panel 
from cell lysates (lane 3). This indicates that SKAP2 could form homodimers with itself.

To assess whether SKAP1 homodimer formation was dependent on the $\mathrm{N}$-terminal domain, a version of Flagtagged SKAP1 with mutations in residues A17/F20/L21 were co-expressed with GFP-tagged wild-type SKAP1 followed by anti-Flag co-precipitation. Mutation of residues A17/F20/L21 abrogated the homodimeric binding of SKAP1 with itself (lane 6). As a control, the blotting of cell lysates showed the expression of the various Flag and GFP tagged proteins (right panel). These data demonstrate the residues in the region of A17 to L21 of the $\mathrm{N}$-terminal region of SKAP1 mediates dimer formation.

We previously showed that SKAP1 binds to RapL and is needed for RapL binding the GTPase Rap1 and the activation of LFA-1 adhesion. N-terminal SKAP1 domain binds to the C-terminal SARAH domain of Rap1. We also show that SKAP1 is needed for RapL binding to membranes in a manner dependent on the PH domain of SKAP1 and the PI3K pathway $[16,17]$. Others have reported other components such as Rap1-dependent integrin regulator Rap1-GTP-interacting adaptor molecule (RIAM) in the multimeric complex [18]. We therefore next asked whether SKAP-1 monomer or dimer formation was needed for SKAP1 binding to RapL (Fig. 2). Tagged wildtype or A17/F20/L21 mutant SKAP1 was co-expressed with RapL in 293T cells and assessed for co-precipitation. While anti-Flag precipitated GFP-tagged SKAP1, the dimer failed to co-precipitate RapL (lane 2). Similarly, anti-GFP coprecipitated Flag-SKAP1 but a faint RapL band (lane 3). For unknown reasons, the Flag-tagged SKAP1 bound to GFP-SKAP1 consistently migrated at a lower Mr suggestive of a post-translational change in

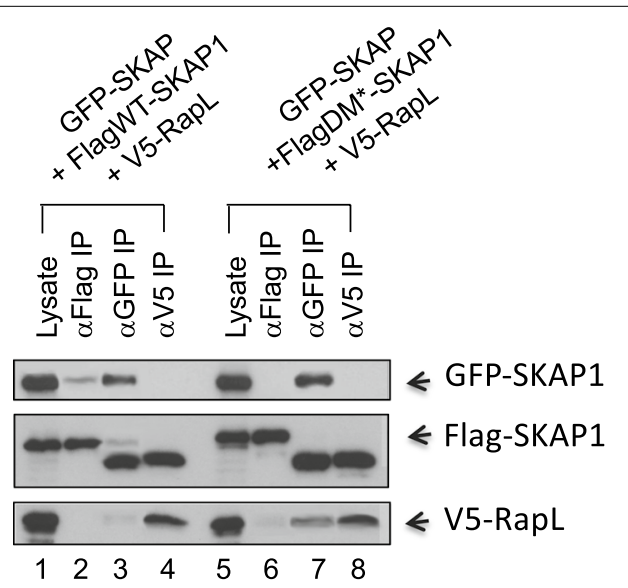

Fig. 2 Dimerisation is not needed for SKAP1 binding to RapL. L293T cells transfected with SKAP-GFP + FlagWT-SKAP1 + V5-Rap (lanes 1-4) or SKAP-GFP + FLAGDM*-SKAP1 + V5-RapL (lanes 5-8). Lane 1,5: lysate; lane 2,6: anti-Flag IP; lane 3,7: anti-GFP IP; lane 4,8: anti-V5 IP the protein. Intriguingly, anti-V5 precipitated V5-tagged RapL with only the lower Mr Flag-tagged SKAP1 (lane 4). This suggests that the lower Mr version of SKAP1 preferentially associates with RapL. Intriguingly, the same patterns of co-precipitation were observed Flag-A17/ F20/L21 SKAP1 (lanes 7 and 8). Attempts were made to transfect primary T-cells for expression but were limited by the low levels of expression that precluded an analysis of binding in these cells. These data indicate that SKAP1 dimer formation is not needed for its binding to RapL.

Lastly, we next showed the presence of the SKAP1 dimer by blotting with anti-SKAP1 rather than antibodies to tags on the proteins (Fig. 3). Combinations of Flag and GFP-tagged SKAP1 were expressed in 293T cells (left panel) and subjected to precipitation using anti-Flag and the followed by blotting with anti-SKAP1 (right panel). Anti-Flag precipitation of Flag SKAP or FlagDM-Skap1 co-precipitated co-expressed GFP-SKAP1 as detected by anti-SKAP-1 (lanes 4 and 5; longer exposure below). We attempted to co-express these vectors in primary mouse T-cells from spleen but were unable to obtain sufficiently high levels of expression of both proteins to carry out similar analysis with these cells. These data confirmed that SKAP1 dimer formation as detected with anti-SKAP1.

\section{Discussion}

Overall, our study shows that SKAP1 forms homodimers dependent on residues A17/F20/L21 in the N-terminus of SKAP1. We previously showed that this region has alternating leucine residues and shares homology with the coiled-coil domain of SKAP-2 [9]. Both SKAP1 and SKAP2 bind ADAP (FYB) through their SH3 domains and served as substrates for the FYN kinase in $\mathrm{T}$ cells $[9,10]$. SKAP1 also colocalizes with another the ADAP binding protein, SLP-76. Mutation of the YDDV sites (termed M12) that disrupt SLP-76 SH2 domain binding interferes with ADAP binding and decreases conjugation and LFA-1 clustering [13]. SKAP1 Src homology 3 (SH3) domains also stabilizes SLP-76 micro-clusters [19].

By contrast, the N-terminal region of SKAP1 binds to RapL such that a RapL mutation (L224A) abrogates SKAP1 binding and arrests T-cells even in the absence of antigen [17]. We now extend these findings by showing that dimerization is not required for the direct binding of SKAP1 to RapL. In fact, the A17/F20/L21 mutant often bound more to RapL than did wild-type SKAP-1. In this context, it is possible that dimerization limits SKAP1 binding to RapL. This contrasts with the reported requirement for the SKAP dimerization for binding to RIAM [19]. It is possible that dimerization may act on functions that are distinct from SKAP1-RapL activation of LFA-1. In the same manner, while the expression of the 

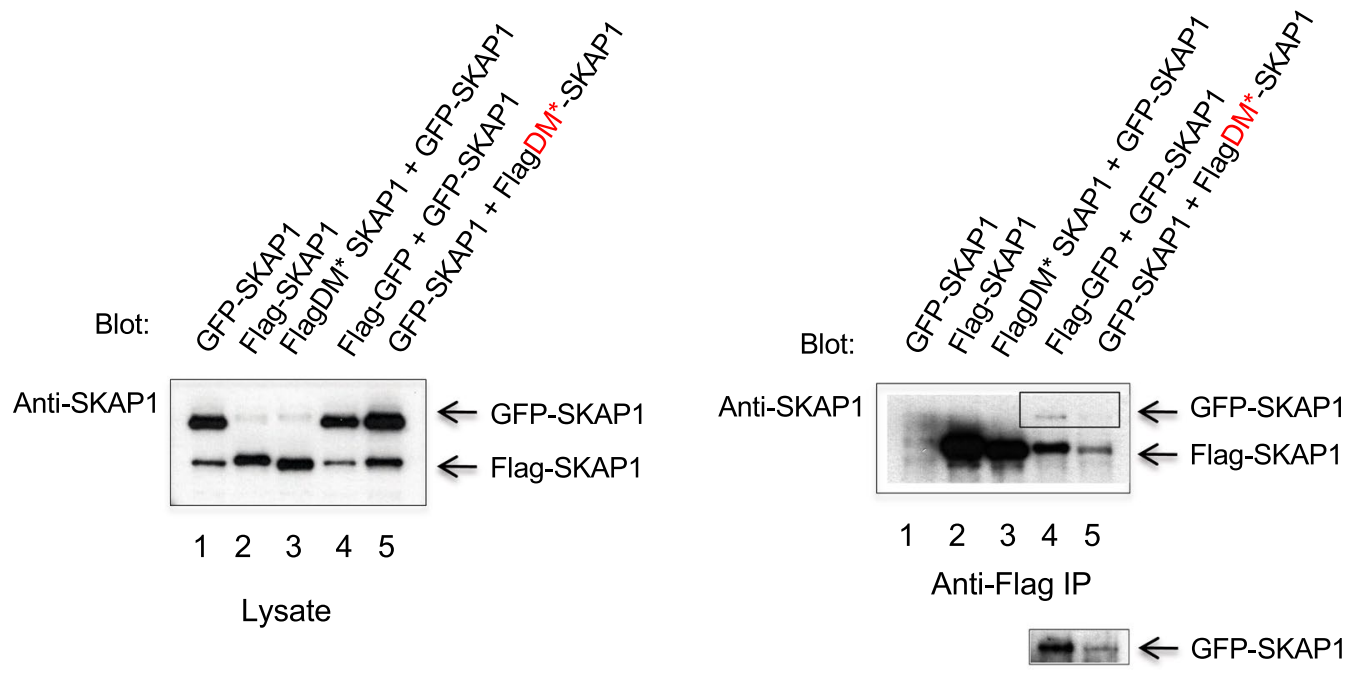

Longer exposure time

Fig. 3 Confirmation of dimerisation with anti-SKAP1 blotting. L293T cells transfected with GFP-SKAP (lane 1), Flag-SKAP1 (lane 2), FlagDM*SKAP1 + Flag SKAP1 (lanes 3), Flag-SKAP1 and SKAP-GFP + (lanes 4) and GFP-SKAP1 + FlagDM*SKAP1 (lanes 5) (left panel). Right panel: anti-Flag precipitates from lysates in left panel. Lower insert shows a longer exposure of a section of lanes 4 and 5

related protein SKAP-55R failed to compensate for the loss of SKAP1 in LFA-1 clustering in mouse T-cell hybridomas [20], it appears to substitute for SKAP1 in stabilizing the formation of microclusters in Jukat T-cells [19]. The full range of functions mediated by SKAP1 dimerization remain to be demonstrated in future studies.

\section{Limitations}

Work restricted to non-lymphoid cells.

\section{Abbreviations}

SLP-76: SH2 domain containing leukocyte protein of $76 \mathrm{kDa}$; PLCY1: phospholipase CY1 (PLCY1); SAM: sterile-a motif; ADAP: adhesion and degranulationpromoting adapter protein; HPK-1: hematopoietic progenitor kinase-1.

\section{Authors' contributions}

$M R, K S$ and $C R$ designed different aspects of the research. MR conducted the experiments. MR, KS and CR drafted the manuscript. All authors read and approved the final manuscript.

\section{Author details}

${ }^{1}$ Department of Obstetrics and Gynaecology, School of Medicine, J.W. Goethe-University, Theodor-Stern-Kai 7, 60590 Frankfurt, Germany. ${ }^{2}$ German Cancer Consortium (DKTK)/German Cancer Research Center, Heidelberg, Germany. ${ }^{3}$ Research Center-Maisonneuve-Rosemont Hospital (CRHMR), Montreal QC H1T 2M4, Canada. ${ }^{4}$ Cell Signalling Section, Department of Pathology, Cambridge University, Cambridge CB2 1QP, UK.

\section{Acknowledgements}

We thank the Department of Obstetrics and Gynaecology, School of Medicine, J.W. Goethe-University and Research Center-Maisonneuve-Rosemont Hospital for technical and administrative support.

\section{Competing interests}

The authors declare that they have no competing interests.

\section{Availability of data and materials}

All relevant material will be freely available to any scientist wishing to use them for non-commercial purposes. Data related to the tables, graph and calculation are available from the corresponding author upon request.

\section{Consent to publish}

Not applicable.

Ethics approval and consent to participate

Not applicable. No human cells from patients.

\section{Funding}

CER was supported by Wellcome Trust Grant (092627/Z/10/Z). MR and KS were supported by Grant BMBF 01GS0850. Funding from both agencies was instrumental in providing support for design and execution of the technical work as well as the writing of the manuscript.

\section{Publisher's Note}

Springer Nature remains neutral with regard to jurisdictional claims in published maps and institutional affiliations.

Received: 8 September 2018 Accepted: 4 December 2018

Published online: 06 December 2018

\section{References}

1. Rudd CE. Adaptors and molecular scaffolds in immune cell signaling. Cell. 1999;96:5-8.

2. Samelson LE. Signal transduction mediated by the T cell antigen receptor: the role of adapter proteins. Annu Rev Immunol. 2002;20:371-94.

3. Houtman JC, Barda-Saad M, Samelson LE. Examining multiprotein signaling complexes from all angles. FEBS J. 2005;2005(272):5426-35.

4. Jordan MS, Singer AL, Koretzky GA. Adaptors as central mediators of signal transduction in immune cells. Nat Immunol. 2003;4:110-6.

5. Yablonski D, Kuhne MR, KadlecekT, Weiss A. Uncoupling of nonreceptor tyrosine kinases from PLC-gamma1 in an SLP-76-deficient T cell. Science. 1998;281:413-6. 
6. da Silva AJ, Li Z, de Vera C, Canto E, Findell P, Rudd CE. Cloning of a novel T-cell protein $\mathrm{FYB}$ that binds FYN and $\mathrm{SH}$ 2-domain-containing leukocyte protein 76 and modulates interleukin 2 production. Proc Natl Acad Sci USA. 1997;94:7493-8.

7. Musci MA, Hendricks-Taylor LR, Motto DG, Paskind M, Kamens J, Turck CW, Koretzky GA. Molecular cloning of SLAP-130, an SLP-76-associated substrate of the $T$ cell antigen receptor-stimulated protein tyrosine kinases. J Biol Chem. 1997;272:11674-7.

8. Di Bartolo V, Montagne B, Salek M, Jungwirth B, Carrette F, Fourtane J, Sol-Foulon N, Michel F, Schwartz O, Lehmann WD, Acuto O. A novel pathway down-modulating $T$ cell activation involves HPK-1-dependent recruitment of 14-3-3 proteins on SLP-76. J Exp Med. 2007;204:681-91.

9. Liu J, Kang H, Raab M, da Silva AJ, Kraeft SK, Rudd CE. FYB (FYN binding protein) serves as a binding partner for lymphoid protein and FYN kinase substrate SKAP55 and a SKAP55-related protein in T cells. Proc Natl Acad Sci USA. 1998;95:8779-84.

10. Marie-Cardine A, Bruyns E, Eckerskorn C, Kirchgessner H, Meuer SC, Schraven B. Molecular cloning of SKAP55, a novel protein that associates with the protein tyrosine kinase p59fyn in human T-lymphocytes. J Biol. 1997;272:16077-80

11. Kang H, Freund C, Duke-Cohan JS, Musacchio A, Wagner G, Rudd CE. SH3 domain recognition of a proline-independent tyrosine-based RKxxYxxY motif in immune cell adaptor SKAP55. EMBO J. 2000;19:2889-99.

12. Kliche S, Breitling D, Togni M, Pusch R, Heuer K, Wang X, Freund C, Kasirer-Friede A, Menasche G, Koretzky GA, Schraven B. The ADAP/ SKAP55 signaling module regulates T-cell receptor-mediated integrin activation through plasma membrane targeting of Rap1. Mol Cell Biol. 2006;26:7130-44.
13. Wang H, Moon EY, Azouz A, Wu X, Smith A, Schneider H, Hogg N, Rudd CE. SKAP-55 regulates integrin adhesion and formation of T cell-APC conjugates. Nat Immunol. 2003;4:366-74.

14. Wang H, Liu H, Lu Y, Lovatt M, Wei B, Rudd CE. Functional defects of SKAP55-deficient T cells identify a regulatory role for the adaptor in LFA-1 adhesion. Mol Cell Biol. 2007;27:6863-75.

15. Wang H, Rudd CE. SKAP-55, SKAP-55-related and ADAP adaptors modulate integrin-mediated immune-cell adhesion. Trends Cell Biol. 2008;18:486-93.

16. Raab M, Smith X, Matthes Y, Strebhardt K, Rudd CE. SKAP1 PH domain determines RAPL membrane localization and Rap1 complex formation for TCR activation of LFA-1. J Biol Chem. 2011;286:29663-70286.

17. Raab M, Wang H, Lu Y, Smith X, Wu Z, Strebhardt K, Ladbury JE, Rudd CE. T cell receptor "inside-out" pathway via signaling module SKAP1-RapL regulates T cell motility and interactions in lymph nodes. Immunity. 2010;32:541-56

18. Raab M, Lu Y, Kohler K, Smith X, Strebhardt K, Rudd CE. LFA-1 activates focal adhesion kinases FAK1/PYK2 to generate LAT-GRB2-SKAP1 complexes that terminate T-cell conjugate formation. Nat Commun. 2017:8:16001.

19. Ophir MJ, Liu BC, Bunnell SC. The N terminus of SKAP55 enables T cell adhesion to TCR and integrin ligands via distinct mechanisms. J Cell Biol. 2013;203:1021-41.

20. Jo EK, Wang H, Rudd CE. An essential role for SKAP-55 in LFA-1 clustering on T cells that cannot be substituted by SKAP-55R. J Exp Med. 2005;201:1733-9.
Ready to submit your research? Choose BMC and benefit from:

- fast, convenient online submission

- thorough peer review by experienced researchers in your field

- rapid publication on acceptance

- support for research data, including large and complex data types

- gold Open Access which fosters wider collaboration and increased citations

- maximum visibility for your research: over 100M website views per year

At BMC, research is always in progress.

Learn more biomedcentral.com/submissions 